PROCEEDINGS OF THE

AMERICAN MATHEMATICAL SOCIETY

Volume 125, Number 11, November 1997, Pages 3177-3183

S 0002-9939(97)03964-6

\title{
EXPONENTIATION IN POWER SERIES FIELDS
}

\author{
FRANZ-VIKTOR KUHLMANN, SALMA KUHLMANN, AND SAHARON SHELAH \\ (Communicated by Andreas R. Blass)
}

\begin{abstract}
We prove that for no nontrivial ordered abelian group $G$ does the ordered power series field $\mathbb{R}((G))$ admit an exponential, i.e. an isomorphism between its ordered additive group and its ordered multiplicative group of positive elements, but that there is a non-surjective logarithm. For an arbitrary ordered field $k$, no exponential on $k((G))$ is compatible, that is, induces an exponential on $k$ through the residue map. This is proved by showing that certain functional equations for lexicographic powers of ordered sets are not solvable.
\end{abstract}

\section{INTRODUCTION}

Let $k$ be an ordered field and $G$ a nontrivial ordered abelian group. Then the ("generalized") power series field $K=k((G))$ admits at least one nonarchimedean order. Further, $K$ is real closed if and only if $k$ is real closed and $G$ is divisible. This provides a very simple and elegant method of constructing nonarchimedean ordered real closed fields. On the other hand, power series fields were already studied by Levi-Civita [LC], later also by H. Hahn $[\mathrm{H}]$, A. Robinson [R] and many others, in an attempt to develop function theory over nonarchimedean fields. One of the first concerns was to define elementary functions (e.g. the logarithm log, or equivalently, its inverse exp) on those fields. It was already known to Levi-Civita that $\log$ is definable through its Taylor expansion on the positive units of $\mathbb{R}((G))$, for archimedean ordered $G$ (cf. the discussion in [L1], [L2]). This was generalized to arbitrary $G$ by B. H. Neumann $[\mathrm{N}]$. But the problem of defining a logarithm from the group $K^{>0}$ of positive elements onto $K$ remained open. We answer this problem in the negative (Theorem 1). In fact, we show that the domain of the logarithm can be extended to $K^{>0}$ if $G$ is divisible, but that a logarithm on $K^{>0}$ will never be surjective onto $K$.

For an exponential $f$ on the ordered field $(K,<)$ we only require $f$ to be an isomorphism between its ordered additive group $(K,+, 0,<)$ and its ordered multiplicative group $\left(K^{>0}, \cdot, 1,<\right)$ of positive elements. If $K=k((G))$, then we

Received by the editors January 31, 1996 and, in revised form, May 18, 1996 .

1991 Mathematics Subject Classification. Primary 12J15, 06A05; Secondary 12J25, 06F20.

Key words and phrases. Ordered exponential fields, power series fields, lexicographic products, convex valuations.

The second author was supported by a Deutsche Forschungsgemeinschaft fellowship. The third author was partially supported by the Edmund Landau Center for research in Mathematical Analysis, and supported by the Minerva Foundation (Germany). Publication number 601. 
say that $f$ is compatible if it induces an exponential on $k$ through the canonical residue map (see Section 2 for details). We shall prove:

Theorem 1. Let $k$ be an ordered field and $G$ a nontrivial ordered abelian group. Let $<$ be any order on $K=k((G))$. Then $(K,<)$ does not admit any compatible exponential. If $(k,<)$ is archimedean, then $(K,<)$ admits no exponential at all.

Theorem 1 shows that the construction method for real closed fields described above is not available for exponential fields. Note that there is an exponential on the surreal numbers (cf. [G]), but this "power series field" is a proper class. For an even stronger version of Theorem 1 , see Theorem 8 .

The key to our result is the fact that every group complement of the valuation ring in $K=k((G))$ is a lexicographic product of ordered abelian groups. Let us recall the definition of lexicographic products. Let $\Gamma$ and $\Delta_{\gamma}, \gamma \in \Gamma$, be totally ordered sets. For every $\gamma \in \Gamma$, we fix a distinguished element $0 \in \Delta_{\gamma}$. The support of $a=\left(\delta_{\gamma}\right)_{\gamma \in \Gamma} \in \prod_{\gamma \in \Gamma} \Delta_{\gamma}$, denoted by $\operatorname{supp}(a)$, is the set of all $\gamma \in \Gamma$ for which $\delta_{\gamma} \neq 0$. As a set, we define $\mathbf{H}_{\gamma \in \Gamma} \Delta_{\gamma}$ to consist of all $\left(\delta_{\gamma}\right)_{\gamma \in \Gamma}$ with well ordered support. The lexicographic order on $\mathbf{H}_{\gamma \in \Gamma} \Delta_{\gamma}$ is introduced as follows. Given $a$ and $b=\left(\delta_{\gamma}^{\prime}\right)_{\gamma \in \Gamma} \in \mathbf{H}_{\gamma \in \Gamma} \Delta_{\gamma}$, observe that $\operatorname{supp}(a) \cup \operatorname{supp}(b)$ is well ordered. Let $\gamma_{0}$ be the least of all elements $\gamma \in \operatorname{supp}(a) \cup \operatorname{supp}(b)$ for which $\delta_{\gamma} \neq \delta_{\gamma}^{\prime}$. We set $a<b: \Leftrightarrow \delta_{\gamma_{0}}<\delta_{\gamma_{0}}^{\prime}$. Then $\left(\mathbf{H}_{\gamma \in \Gamma} \Delta_{\gamma},<\right)$ is a totally ordered set, the lexicographic product of the ordered sets $\Delta_{\gamma}$. If $\Delta_{\gamma}=\Delta$ for all $\gamma \in \Gamma$ then we write $\Delta^{\Gamma}$ for their lexicographic product; it consists of all maps from $\Gamma$ to $\Delta$ with well ordered support.

If all $\Delta_{\gamma}$ are totally ordered abelian groups, then we can take the distinguished elements 0 to be the neutral elements of the groups $\Delta_{\gamma}$. Defining addition on $\mathbf{H}_{\gamma \in \Gamma} \Delta_{\gamma}$ componentwise, we obtain a totally ordered abelian group

$$
\left(\mathbf{H}_{\gamma \in \Gamma} \Delta_{\gamma},+, 0,<\right)
$$

the Hahn product of the ordered groups $\Delta_{\gamma}$.

In Section 3, we prove the following theorem and explain how it relates to the surjectivity of a logarithm.

Theorem 2. Let $\Gamma$ and $\Delta$ be totally ordered sets without greatest element, and fix an element $0 \in \Delta$. Suppose that $\Gamma^{\prime}$ is a cofinal subset of $\Gamma$ and that $\iota: \Gamma^{\prime} \rightarrow \Delta^{\Gamma}$ is an order preserving embedding. Then the image $\iota \Gamma^{\prime}$ is not convex in $\Delta^{\Gamma}$.

The same holds for an order preserving embedding $\iota: \Gamma^{\prime} \rightarrow \mathbf{H}_{\gamma \in \Gamma} \Delta_{\gamma}$ and already under the condition that $\Gamma$ has no greatest element and 0 is not the greatest element of $\Delta_{\gamma}$ for any $\gamma \in \Gamma$ (cf. [K-K-S]). If we drop the condition that $\Gamma$ has no greatest element, the situation changes drastically. Suitably chosen ordered sets $\Gamma$ and $\Delta$ will even admit an isomorphism $\Gamma \simeq \Delta^{\Gamma}$. We study this situation and related questions in $[\mathrm{K}-\mathrm{K}-\mathrm{S}]$.

\section{Preliminaries on left Logarithms}

Let $G$ be a totally ordered abelian group. The set of archimedean classes $[a]$ of nonzero elements $a \in G$ is totally ordered by setting $[a]<[b]$ if $|a| \gg|b|$. The map $v: a \mapsto[a]$ is called the natural valuation of $G$. It satisfies the triangle inequality $v(a-b) \geq \min \{v a, v b\}$ and $v(-a)=v a$ as well as

$$
a \leq b \leq 0 \vee a \geq b \geq 0 \Rightarrow v a \leq v b .
$$


In this paper, $(K,<)$ will always be a totally ordered field. We let $v$ denote the natural valuation on its additive group $(K,+, 0,<)$. In this case, $v K:=$ $v(K \backslash\{0\})$ forms a totally ordered abelian group endowed with the addition $[a]+$ $[b]:=[a b]$, and $v$ is a field valuation. For more information on natural valuations, see $[\mathrm{K}]$.

Let $w$ be any field valuation on $K$. The value group of $(K, w)$ will be denoted by $w K$ and its residue field by $K w$. Further, $w$ is convex with respect to $<$ if it satisfies (1). The valuation ring $R_{w}=\{a \in K \mid w a \geq 0\}$ of a convex valuation $w$ is convex in $K$, and so is its valuation ideal $I_{w}=\{a \in K \mid w a>0\}$. Further, the set $\mathcal{U}_{w}^{>0}:=\{a \in K \mid w a=0 \wedge a>0\}$ of positive units of $R_{w}$ is a convex subgroup of $\left(K^{>0}, \cdot, 1,<\right)$. Note that $w$ is convex if and only if $R_{v} \subseteq R_{w}$ (i.e., $w$ is a coarsening of $v$ ), in which case its value group $w K$ is the quotient of $v K$ by a convex subgroup.

If $K$ admits an exponential, then its multiplicative group of positive elements is divisible (since the additive is). In order to prove Theorem 1, we can thus always assume divisibility. As in [K] (Lemma 3.4 and Theorem 3.8), we then have the following representations as lexicographic sums:

$$
(K,+, 0,<) \simeq \mathbf{A}_{w} \amalg\left(R_{w},+, 0,<\right)
$$

where $\mathbf{A}_{w}$ is an arbitrary group complement of $R_{w}$ in $(K,+)$, and analogously,

$$
\left(K^{>0}, \cdot, 1,<\right) \simeq \mathbf{B}_{w} \amalg\left(\mathcal{U}_{w}^{>0}, \cdot, 1,<\right)
$$

where $\mathbf{B}_{w}$ is an arbitrary group complement of $\mathcal{U}_{w}^{>0}$ in $\left(K^{>0}, \cdot\right)$. Endowed with the restriction of the ordering, $\mathbf{A}_{w}$ and $\mathbf{B}_{w}$ are unique up to isomorphism. In view of (1) and the fact that $w(-a)=w a$, the map

$$
-w:\left(K^{>0}, \cdot, 1,<\right) \rightarrow(w K,+, 0,<), \quad-w a=w a^{-1}
$$

is a surjective group homomorphism preserving $\leq$, with kernel $\mathcal{U}_{w}^{>0}$. We find that every complement $\mathbf{B}_{w}$ is isomorphic to $(w K,+, 0,<)$ through the map $-w$.

An exponential $f$ on $K$ will be called compatible with $w$ if it satisfies that $f\left(R_{w}\right)=\mathcal{U}_{w}^{>0}$ and $f\left(I_{w}\right)=1+I_{w}$. Since $K w=R_{w} / I_{w}$ and $\left(K w^{>0}, \cdot, 1,<\right)$ $=\left(\mathcal{U}_{w}^{>0}, \cdot, 1,<\right) / 1+I_{w}$, this means that $f$ induces canonically an exponential $f w:(K w,+, 0,<) \rightarrow\left(K w^{>0}, \cdot, 1,<\right)$. The canonical valuation $w$ of a power series field $K=k((G))$ has value group $G$ and residue field $k$. Further, it is henselian. Consequently, $w$ is convex with respect to every order $<$ on $K$ (cf. $[\mathrm{KN}-\mathrm{W}])$. Hence, $f$ is compatible on $k((G))$ if and only if it is compatible with $w$.

Remark 3. If an ordered field $K$ admits an exponential, then it admits an exponential which is compatible with the natural valuation (cf. $[\mathrm{K}]$, Section 3.3).

Let $w$ be any convex valuation on $K$. Every compatible exponential $f$ decomposes into two isomorphisms of ordered groups:

$$
\begin{aligned}
f_{R}: & \left(R_{w},+, 0,<\right) & \rightarrow\left(\mathcal{U}_{w}^{>0}, \cdot, 1,<\right), \\
f_{L}: & \mathbf{A}_{w} & \rightarrow \mathbf{B}_{w} .
\end{aligned}
$$

Conversely, in view of (2) and (3), such isomorphisms $f_{R}$ and $f_{L}$ can be put together to obtain an exponential compatible with $w$. The inverse $f_{L}^{-1}$ is called a left logarithm, and $f_{R}^{-1}$ a right logarithm. Through the isomorphism (4), every isomorphism

$$
h:(w K,+, 0,<) \rightarrow \mathbf{A}_{w}
$$


gives rise to a left logarithm $h \circ-w$. Conversely, given a left logarithm $f_{L}^{-1}$, the map $f_{L}^{-1} \circ(-w)^{-1}$ is such an isomorphism $h$. This correspondence motivates the following definition: a logarithmic cross-section of an ordered field $(K,<)$ with respect to a convex valuation $w$ is an order preserving embedding $h$ of $w K$ into an additive group complement of the valuation ring (that is, an embedding $h$ of $w K$ into the additive group $(K,+, 0,<)$ satisfying $w h(g)<0$ for all $g \in w K)$. Thus, every left logarithm induces a logarithmic cross-section which is surjective (i.e., $h(w K)$ is an additive group complement to the valuation ring), and vice-versa.

Our goal in the next section is to show that power series fields always admit logarithmic cross-sections, but never surjective ones.

\section{LEXICOGRAPHIC PRODUCTS AND LOGARITHMIC CROSS-SECTIONS}

Proof of Theorem 2. Assume that $\Gamma$ and $\Delta$ are totally ordered sets, and fix an element $0 \in \Delta$. Assume further that $\Delta$ has no greatest element, so that we can choose a map $\tau: \Delta \rightarrow \Delta$ such that $\tau \delta>\delta$ for all $\delta \in \Delta$. For every well ordered set $S \subset \Gamma$ and every $d=\left(d_{\gamma}\right)_{\gamma \in \Gamma} \in \Delta^{\Gamma}$, set

$$
d \oplus S=\left(d_{\gamma}^{\prime}\right)_{\gamma \in \Gamma} \text { where } d_{\gamma}^{\prime}:=\left\{\begin{array}{cl}
d_{\gamma} & \text { if } \gamma \notin S, \\
\tau d_{\gamma} & \text { if } \gamma \in S .
\end{array}\right.
$$

Observe that the support of $d \oplus S$ is contained in $\operatorname{supp}(d) \cup S$ and thus, it is again well ordered. Further, if $S, S^{\prime} \subset \Gamma$ are well ordered sets (or empty), then

$$
S \varsubsetneqq S^{\prime} \Rightarrow d \oplus S<d \oplus S^{\prime}
$$

Now suppose that $\Gamma$ has no greatest element, $\Gamma^{\prime}$ is a cofinal subset of $\Gamma$ and $\iota: \Gamma^{\prime} \rightarrow \Delta^{\Gamma}$ is an order preserving embedding such that the image $\iota \Gamma^{\prime}$ is convex in $\Delta^{\Gamma}$. We wish to deduce a contradiction.

By induction on $n \in \mathbb{N}$, we define elements $\gamma_{0}^{(n)} \in \Gamma^{\prime}$. We choose an arbitrary $\gamma_{0}^{(1)} \in \Gamma^{\prime}$. Having already constructed $\gamma_{0}^{(n)}$, we carry through the following induction step. Since $\Gamma$ has no greatest element, the same holds for $\Gamma^{\prime}$, and there is some $\alpha^{(n)} \in \Gamma^{\prime}$ such that $\gamma_{0}^{(n)}<\alpha^{(n)}$. Hence, $\iota \gamma_{0}^{(n)}<\iota \alpha^{(n)}$. Let $\beta^{(n)} \in \Gamma$ be the least element of $\operatorname{supp}\left(\iota \gamma_{0}^{(n)}\right) \cup \operatorname{supp}\left(\iota \alpha^{(n)}\right)$ for which

$$
\left(\iota \gamma_{0}^{(n)}\right)_{\beta^{(n)}}<\left(\iota \alpha^{(n)}\right)_{\beta^{(n)}} .
$$

Since $\Gamma$ has no greatest element and $\Gamma^{\prime}$ is a cofinal subset, we can choose $\gamma_{0}^{(n+1)} \in \Gamma^{\prime}$ such that $\beta^{(n)}<\gamma_{0}^{(n+1)}$.

If $S \subset \Gamma$ is a well ordered set with least element $\gamma_{0}^{(n+1)}$, then

$$
\iota \gamma_{0}^{(n)}<\iota \gamma_{0}^{(n)} \oplus S<\iota \alpha^{(n)} .
$$

Indeed, $\left(\iota \gamma_{0}^{(n)} \oplus S\right)_{\beta}=\left(\iota \gamma_{0}^{(n)}\right)_{\beta}$ for every $\beta<\gamma_{0}^{(n+1)}$. In particular,

$$
\left(\iota \gamma_{0}^{(n)} \oplus S\right)_{\beta^{(n)}}=\left(\iota \gamma_{0}^{(n)}\right)_{\beta^{(n)}}<\left(\iota \alpha^{(n)}\right)_{\beta^{(n)}},
$$

which implies the second inequality of (6). Its first inequality follows from (5).

The image of $\Gamma^{\prime}$ in $\Delta^{\Gamma}$ being convex, (6) yields that also $\iota \gamma_{0}^{(n)} \oplus S$ lies in this image. Thus, $\iota^{-1}\left(\iota \gamma_{0}^{(n)} \oplus S\right)$ is a well defined element of $\Gamma^{\prime}$.

Suppose now that for some ordinal number $\mu \geq 1$ we have chosen elements $\gamma_{\nu}^{(n)} \in \Gamma^{\prime}, \nu<\mu, n \in \mathbb{N}$, such that for every fixed $n$, the sequence $\left(\gamma_{\nu}^{(n)}\right)_{\nu<\mu}$ is 
strictly increasing. Then we set

$$
\gamma_{\mu}^{(n)}:=\iota^{-1}\left(\iota \gamma_{0}^{(n)} \oplus\left\{\gamma_{\nu}^{(n+1)} \mid \nu<\mu\right\}\right) \in \Gamma^{\prime}
$$

for every $n \in \mathbb{N}$. If $\lambda<\mu$, then $\left\{\gamma_{\nu}^{(n+1)} \mid \nu<\lambda\right\} \varsubsetneqq\left\{\gamma_{\nu}^{(n+1)} \mid \nu<\mu\right\}$ and thus, $\gamma_{\lambda}^{(n)}<\gamma_{\mu}^{(n)}$ by (5). So for every ordinal number $\mu$, the sequences $\left(\gamma_{\nu}^{(n)}\right)_{\nu<\mu}$ can be extended. We obtain strictly increasing sequences of arbitrary length, contradicting the fact that their length is bounded by the cardinality of $\Gamma^{\prime}$.

Now we apply Theorem 2 to logarithmic cross-sections of the power series field $K=k((G))$ with canonical valuation $w$. One of the complements for the valuation ring $R_{w}=k[[G]]$ is the Hahn product $\mathbf{H}_{G<0}(k,+, 0,<)$, which we will denote by $k^{G^{<0}}$. Since the complements are unique up to isomorphism, a surjective logarithmic cross-section $h$ with respect to $w$ would induce an isomorphism $G \simeq k^{G^{<0}}$. This in turn would imply that $G^{<0}$ has no greatest element and would give rise to an embedding of $G^{<0}$ in $k^{G^{<0}}$ with convex image, which contradicts Theorem 2. So we have proved:

Theorem 4. Let $k$ be an ordered field and $G$ a nontrivial ordered abelian group. Further, let $w$ be the canonical valuation on $K=k((G))$ and $<$ any order on $K$. Then $(K,<)$ admits no surjective logarithmic cross-section with respect to $w$.

This theorem implies Theorem 1. Indeed, a compatible exponential of $K$ would induce a surjective logarithmic cross-section with respect to $w$, which is impossible. If $(k,<)$ is archimedean, then $w$ will coincide with the natural valuation $v$ of $(k((G)),<)$. So the second assertion of Theorem 1 follows by Remark 3 .

If $G$ is an ordered abelian group, then we denote its natural valuation by $v_{G}$. For the definition of the archimedean components $B_{\gamma}$ of $G$ (where $\gamma \in v_{G} G$ ), see [FU]. They are archimedean ordered abelian groups. Hahn's embedding theorem states that there is an order preserving group embedding $\rho$ of $G$ in the Hahn product $\mathbf{H}_{\gamma \in v_{G} G} B_{\gamma}$, if $G$ is divisible (cf. [H] or [FU] , IV, Theorem 16).

Proposition 5. Let $G$ be a nontrivial divisible ordered abelian group. Then $\mathbb{R}((G))$ admits a logarithmic cross-section. If every archimedean component of $G$ embeds in the ordered additive group of $k$, then $k((G))$ admits a logarithmic cross-section.

Proof. By taking representatives, we obtain an embedding $\sigma: v_{G} G \rightarrow G^{<0}$; it is order preserving by (1). Now $\sigma$ lifts to an embedding $\hat{\sigma}: k^{v_{G} G} \rightarrow k^{G^{<0}}$. If every archimedean component $B_{\gamma}$ of $G$ embeds in $(k,+, 0,<)$, then there is an embedding $\tau: \mathbf{H}_{\gamma \in v_{G} G} B_{\gamma} \rightarrow \mathbf{H}_{v_{G} G}(k,+, 0,<)=k^{v_{G} G}$. So $h=\hat{\sigma} \circ \tau \circ \rho$ is the required logarithmic cross-section for $k((G))$. Since every archimedean ordered abelian group embeds in $\mathbb{R}$, the first assertion follows from the second.

Corollary 6. If $G$ is nontrivial and divisible, then the real closed field $K=$ $\mathbb{R}((G))$ admits a non-surjective logarithm, i.e., an embedding $\left(K^{>0}, \cdot, 1,<\right) \rightarrow$ $(K,+, 0,<)$.

For the proof, note that a right logarithm on $\mathbb{R}((G))$ always exists: it is defined on the positive units of the valuation ring $\mathbb{R}[[G]]$ through the logarithmic power series (cf. [A]). In combination with a non-surjective logarithmic cross-section, it gives rise to the desired (non-surjective) logarithm. By taking the union over a suitable countable ascending chain of power series fields with non-surjective logarithms, we can obtain a surjective logarithm. Using this construction, we prove in $[\mathrm{K}-\mathrm{K} 2]$ the 
existence of exponential fields with arbitrary given exponential rank ( $=$ the order type of the set of all convex valuations compatible with the exponential).

Remark 7. Kaplansky $[\mathrm{KA}]$ has shown that a valued field is maximal (i.e., admits no proper immediate extensions) if and only if every pseudo Cauchy sequence admits a limit. The same principle was proved by Fleischer $[\mathrm{F}]$ for valued abelian groups. It can also be proved for certain classes of valued modules. At first sight, one might believe that this principle holds for all (reasonable) valued structures. But the nonarchimedean exponential fields with their natural valuation constitute a counterexample:

There are maximal naturally valued exponential fields (i.e., they do not admit proper immediate extensions to which also the exponential extends). These are precisely the exponential fields whose natural valuation $v$ is complete: On the one hand, it was remarked in $[\mathrm{K}]$ that if $(L, v) \supset(K, v)$ is immediate and the exponential extends from $K$ to $L$, then $(K, v)$ is dense in $(L, v)$. On the other hand, if $(K, v)$ is dense in $(L, v)$, then an exponential of $K$ extends to $L$ by continuity. Hence, the completion of a nonarchimedean exponential field with respect to its natural valuation is the maximal immediate extension as a naturally valued exponential field. But by our nonexistence result, it cannot be a power series field. On the other hand, Kaplansky has also shown in $[\mathrm{KA}]$ that a valued field $(K, w)$ of residue characteristic 0 is a power series field with canonical valuation $w$ if and only if it is maximal. (Note that the natural valuation has residue characteristic 0 since the residue field is ordered.) Hence, a maximal naturally valued exponential field is not maximal as a valued field.

An argument similar to that used in establishing Theorem 4 shows that a Hahn group (i.e. a maximally valued group) cannot be an exponential group in the sense of $[\mathrm{K}]$, and thus cannot be the natural value group of an exponential field. Further consequences of Theorem 2 for exponential groups will be studied in a subsequent paper.

Under the hypothesis of Theorem 1 we can prove that an exponential cannot even be compatible with any nontrivial coarsening $w^{\prime}$ of $w$ : Since $K$ is a power series field with canonical valuation $w$, it can also be written as a power series field $(K w)\left(\left(w^{\prime} K\right)\right)$ with canonical valuation $w^{\prime}$, and from Theorem 1 it follows that no exponential can be compatible with $w^{\prime}$. We have seen in the above remark that we can talk about maximal valuations instead of power series fields. So we can restate our result as follows: If the ordered field $K$ admits an exponential $f$, then there is no nontrivial coarsening of its natural valuation $v$ which is maximal and compatible with $f$. We prove the following generalization:

Theorem 8. Let $f$ be an exponential on the ordered field $K$ and $w$ a coarsening of the natural valuation $v$ of $K$ such that $f$ is compatible with $w$. Then there is no coarsening $\tilde{w}$ of $w$ such that the valuation $\bar{w}=w / \tilde{w}$ induced by $w$ on the residue field $K \tilde{w}$ is nontrivial and $(K \tilde{w}, \bar{w})$ is maximal.

Proof. Suppose to the contrary that such a coarsening $\tilde{w}$ exists. We have that $R_{w} \subset R_{\tilde{w}}$. Let $\overline{\mathbf{A}}$ be a group complement of $R_{w}$ in $R_{\tilde{w}}$ and $\tilde{\mathbf{A}}$ a group complement of $R_{\tilde{w}}$ in $(K,+, 0,<)$. Then $\tilde{\mathbf{A}} \amalg \overline{\mathbf{A}}$ is a group complement of $R_{w}$ in $(K,+, 0,<)$. Further, $f$ induces an isomorphism $h$ from $G=w K$ onto $\tilde{\mathbf{A}} \amalg \overline{\mathbf{A}}$ as ordered groups. In particular, $G^{<0}$ has no greatest element. 
The value group of $\bar{w}$ is isomorphic to a nontrivial convex subgroup $\bar{G}$ of $G$. Since $(K \tilde{w}, \bar{w})$ is maximal and has residue field $(K \tilde{w}) w / \tilde{w}=K w$, it is isomorphic to the power series field $(K w)((\bar{G}))$. Hence, $\overline{\mathbf{A}}$ is isomorphic to a Hahn product $(K w)^{\bar{G}^{<0}}$. This yields an embedding of the nontrivial convex subgroup $H:=\bar{G} \cap h^{-1}(\overline{\mathbf{A}})$ of $\bar{G}$ in $(K w)^{G^{<0}}$. Under this embedding, the image of the final segment $H^{<0}$ of $\bar{G}<0$ is convex in $(K w)^{\bar{G}^{<0}}$. But $\bar{G}^{<0}$ is a final segment of $G^{<0}$ and thus has no greatest element. This contradicts Theorem 2 .

\section{REFERENCES}

[A] Alling, N. L. On exponentially closed fields, Proc. A.M.S. 13 No. 5 (1962), 706-711. MR 25:5058

[F] Fleischer, I. Maximality and ultracompleteness in normed modules, Proc. A.M.S. 9 (1958), 151-157. MR 20:216

[FU] Fuchs, L. Partially ordered algebraic systems, Pergamon Press, Oxford (1963). MR 30:2090

[G] Gonshor, H. An introduction to the theory of surreal numbers, Cambridge University Press (1986). MR 88b:04002

[H] Hahn, H. Über die nichtarchimedischen Größensysteme, S.-B. Akad. Wiss. Wien, math.naturw. Kl. Abt. IIa, 116 (1907), 601-655.

[KA] Kaplansky, I. Maximal fields with valuations I, Duke Math. Journ. 9 (1942), 303-321. MR 3:264d

[KN-W] Knebusch, M. - Wright, M. Bewertungen mit reeller Henselisierung, J. reine angew. Math. 286/287 (1976), 314-321. MR 54:7440

[K] Kuhlmann, S. On the structure of nonarchimedean exponential fields I, Archive for Math. Logic 34 (1995), 145-182.

[K-K1] Kuhlmann, F.-V. - Kuhlmann, S. On the structure of nonarchimedean exponential fields II, Comm. in Algebra 22(12) (1994), 5079-5103.

[K-K2] Kuhlmann, F.-V. - Kuhlmann, S. The exponential rank of nonarchimedean exponential fields, preprint.

[K-K-S] Kuhlmann, F.-V. - Kuhlmann, S. - Shelah, S. Functional equations for lexicographic products, preprint.

[L1] Laugwitz, D. Eine nichtarchimedische Erweiterung angeordneter Körper, Math. Nachr. 37 (1968), 225-236. MR 39:4125

[L2] Laugwitz, D. Tullio Levi-Civita's work on nonarchimedean structures, in: Tullio LeviCivita, Convegno internazionale celebrativo del centenario della nascita, Academia Nazionale dei Lincei, Atti dei Convegni Lincei 8 (1973), 297-312.

[LC] Levi-Civita, T. Sugli infiniti ed infinitesimi attuali quali elementi analitici (1892-1893), opere mathematiche, vol. 1, Bologna (1954), 1-39.

[N] Neumann, B. H. On ordered division rings, Trans. A.M.S. 66 (1949), 202-252. MR 11:311f

[R] Robinson, A. Function theory on some nonarchimedean fields, Amer. Math. Monthly 80 (1973), 87-109. MR 48:8464

Mathematisches Institut der Universität Heidelberg, Im Neuenheimer Feld 288, D69120 Heidelberg, Germany

E-mail address: fvk@harmless.mathi.uni-heidelberg.de

Department of Mathematics, The Hebrew University of Jerusalem, Jerusalem, Israel

E-mail address: shelah@sunrise.huji.ac.il 